\title{
Nucleon Resonances: From Photoproduction to High Photon Virtualities
}

Received: 23 August 2016 / Accepted: 24 August 2016 / Published online: 22 September 2016 (C) Springer-Verlag Wien 2016

The topical workshop "Nucleon Resonances: From Photoproduction to High Photon Virtualities" took place at the European Center for Theoretical Studies in Nuclear Physics and Related Areas in Trento, Italy from October 12-16, 2015. The organizing committee consisted of R.W. Gothe (Chair, USC), V.I. Mokeev (Jefferson Lab), and E. Santopinto (INFN).

The Workshop had around 40 participants and was supported in part also by generous contributions from JSA/Jefferson Lab, the University of South Carolina, and INFN.

The studies of the excited nucleon $\mathrm{N}^{*}$ spectrum and the structure of these states offer unique information on many facets of the non-perturbative strong interaction in the generation of excited nucleon states of different quantum numbers. The workshop was devoted to the study of the spectrum and structure of $\mathrm{N}^{*}$ states, as they become accessible through the electromagnetic excitation of the nucleon in exclusive meson production. It was focused on: (a) the baryon spectrum in exclusive meson photoproduction, (b) the search for new baryon states in the combined studies of exclusive photo- and electroproduction at small and moderate photon virtualities $\left(\mathrm{Q}^{2}\right)$, (c) electro-excited $\mathrm{N}^{*}$ states and their structure for $\mathrm{Q}^{2}$ up to $5 \mathrm{GeV}^{2}$, and (d) the extension of these studies in the future experiments with CLAS12 at Jefferson Lab up to $12 \mathrm{GeV}^{2}$.

The current status of the studies of the excited nucleon spectrum and structure from exclusive meson photoand electroproduction data, as well as the challenges and prospects in this field, such as the search for new states of baryon matter, the so-called hybrid baryons, with glue as an extra structural component, were reviewed in a keynote talk by V.D. Burkert (JLab), which helped to shape the Workshop discussions and outcome.

The experimental studies of exclusive meson photoproduction off the nucleon at the JLab/CLAS, ELSA, and MAMI facilities continue their rapid progress. The results were presented in talks by S. Strauch (USC) and A. D'Angelo (Rome U.). These experiments provided detailed information on all exclusive meson photoproduction channels relevant in the resonance region, including differential cross sections, and single, double, and triple polarization asymmetries. The data on exclusive photoproduction off the nucleon with more than one meson in the final states keeps growing. The status and prospects of the advanced reaction models for the extraction of the resonance parameters from these data were presented in talks by L. Tiator (Mainz U.),

This preface belongs to the special issue "Nucleon Resonances".

Ralf W. Gothe

Department of Physics and Astronomy, University of South Carolina, 712 Main Street, Columbia, SC 29208, USA

E-mail: gothe@sc.edu

Viktor Mokeev

Jefferson Lab’ 1200 Jefferson Ave Suite 5, Newport News, VA 23606, USA

E-mail: mokeev@jlab.org

Elena Santopinto $(\varangle)$

Istituto Nazionale di Fisica Nucleare, via Dodecaneso 33, 16146 Genova, Italy

E-mail: elena.santopinto@ge.infn.it 
I. Danilkin (JPAC at JLab), H. Haberzettl (GWU), J. Nys (Ghent U.), A. Sarantsev (Bonn U. and Petersburg Nucl. Phys. Inst.), and H. Kamano (Osaka U.). Analyses of the aforementioned experimental data considerably extended our knowledge of the $\mathrm{N}^{*}$ spectrum. Several candidate $\mathrm{N}^{*}$ states were included to the 2014 edition of the PDG as an outcome of these efforts with decisive contribution of the KY photoproduction data from $\mathrm{Jlab}$ /CLAS and ELSA carried out within the framework of global multi-channel Bonn-Gatchina approach. The search for manifestations of these new $\mathrm{N}^{*}$ states in the future exclusive electroproduction data at low $\mathrm{Q}^{2}$ will be the critical step to prove or rule out their existence.

The CLAS detector at JLab has produced the dominant part of the available worldwide data on all relevant meson electroproduction channels off the nucleon in the resonance region for $\mathrm{Q}^{2}$ up to $5.0 \mathrm{GeV}^{2}$. The recent results from these studies were presented in talks by K. Park (JLab) and D.S. Carman (JLab). Analyses of the data on $N \pi, N \eta$, and $\pi^{+} \pi^{-}$p exclusive electroproduction off the proton have provided the only worldwide results available on the $\mathrm{Q}^{2}$ evolution of the helicity amplitudes for the transitions from the initial photonproton to the final $\mathrm{N}^{*}$ states, the so-called $\gamma_{\mathrm{v}} \mathrm{NN}^{*}$ electrocouplings, which allow us to explore the $\mathrm{N}^{*}$ internal structure. The efforts on the extraction of the $\gamma_{\mathrm{v}} \mathrm{NN}^{*}$ electrocouplings were reviewed in the talk by V.I. Mokeev (JLab). $\gamma_{\mathrm{v}} \mathrm{NN}^{*}$ electrocouplings have become available for most excited nucleon states in the mass range up to $1.8 \mathrm{GeV}$ at photon virtualities up to $5.0 \mathrm{GeV}^{2}$ (up to $7.5 \mathrm{GeV}^{2}$ for $\Delta(1232) 3 / 2^{+}$and $7.0 \mathrm{GeV}^{2}$ for $\left.\mathrm{N}(1535) 1 / 2^{-}\right)$. The current results on the resonance electrocouplings have been collected at: https://userweb. jlab.org/ mokeev/resonance_electrocouplings/. Physics analyses of these results have revealed the structure of $\mathrm{N}^{*}$ states for $\mathrm{Q}^{2}<5.0 \mathrm{GeV}^{2}$ as a complex interplay between the inner core of three dressed quarks and the external meson-baryon cloud.

For the first time results on exclusive $\pi^{-}$p electroproduction off bound neutrons corrected for kinematical final-state-interactions and Fermi-motion have become available and were presented in the talk by R.W. Gothe (USC). The impressive progress in advanced reaction models allowing us to account for the $\pi^{-} \mathrm{p}$ final-stateinteractions and Fermi-motion of the neutron-target inside the deuteron, presented in the talk by T-S.H. Lee (ANL), opens up the prospects for extracting the $\gamma_{\mathrm{v}} \mathrm{NN}^{*}$ electrocouplings off the neutron.

The development of theoretical approaches capable of relating the non-perturbative strong interaction mechanisms behind the formation of $\mathrm{N}^{*}$ states to the results on the $\mathrm{N}^{*}$ spectrum and the $\gamma_{\mathrm{v}} \mathrm{NN}^{*}$ electrocouplings, reviewed in the colloquium talk by C.D. Roberts (ANL) and the presentation by V.M. Braun (Regensburg U.), represent a key part in the synergetic efforts between experimentalists and theorists in the studies of $\mathrm{N}^{*}$ states.

The Dyson-Schwinger Equations of QCD (DSEQCD) successfully reproduce the data on elastic and transition $\mathrm{N} \rightarrow \Delta(1232) 3 / 2^{+}, \mathrm{N} \rightarrow \mathrm{N}(1440) 1 / 2^{+}$form factors for $\mathrm{Q}^{2}>2.5 \mathrm{GeV}^{2}$ with the same dressed quark mass function, demonstrating the relevance of dressed quarks in the structure of the ground and excited nucleons and the capability of accessing this fundamental quantity from the data on the nucleon elastic and transition $\mathrm{N} \rightarrow \mathrm{N}^{*}$ form factors. Impressive developments in the studies of the $\mathrm{N}^{*}$ spectrum and structure within DSEQCD were presented in the talk by J. Segovia (Technical U of Munich), G. Eichmann (Giessen U.), S. Qin (ANL), B. El-Bennich (Sao Paulo U.), A. Bashir (Michoacán U.), P. Rodriguez-Quintero (Huelva U.), and D. Binosi (ECT*).

The novel approach presented in the talk by N. Offen (Regensburg U.) provides insight into the $\mathrm{N}^{*}$ partonic structure, allowing us to constrain the quark distribution amplitudes of the $\mathrm{N}(1535) 1 / 2^{-}$resonance, relating them to the resonance electrocouplings determined by employing Light Cone Sum Rules. The moments of the quark distribution amplitudes can be computed from the QCD Lagrangian within LQCD and confronted to those derived from the $\gamma_{\mathrm{v}} \mathrm{NN}^{*}$ electrocoupling values offering an additional promising avenue in relating empirical resonance electrocoupling values from experiment to the first principles of QCD.

Plans for the evaluation of the $\gamma_{\mathrm{v}} \mathrm{NN}^{*}$ electrocouplings at intermediate photon virtualities from the QCD Lagrangian within the LQCD framework were presented in talks by D.G. Richards (JLab) and R. Briceno (JLab).

The constituent quark models remain the only available tool in the studies of the $\mathrm{N}^{*}$ structure over the full $\mathrm{N}^{*}$ spectrum. The recent results of the advanced quark models were presented in talks by G. de Teramond (Univ. of Costa Rica), I.T. Obukhovsky (Moscow State U.), G. Ramalho (Univ. of Rio Grande), H. Garcia (INFN), J. Feretti (INFN), Y. Yamaguchi (INFN), M.M. Giannini (Univ. of Genova), and E. Santopinto (INFN). New results on resonance electrocouplings for $\mathrm{Q}^{2}<12 \mathrm{GeV}^{2}$ from these promising approaches are urgently needed.

After completion of the Jefferson Lab $12 \mathrm{GeV}$ Upgrade, CLAS12 will be the only facility foreseen worldwide capable of exploring the $\gamma_{\mathrm{v}} \mathrm{NN}^{*}$ electrocouplings at the smallest $0.05 \mathrm{GeV}^{2}<\mathrm{Q}^{2}<0.3 \mathrm{GeV}^{2}$ and at the highest $\mathrm{Q}^{2}$ up to $12 \mathrm{GeV}^{2}$ ever achieved in exclusive reactions. The search for new types of baryon matter, the so-called hybrid baryons with glue as a structural component, represents a new flagship experiment 
in the $\mathrm{N}^{*}$ studies at small/intermediate $\mathrm{Q}^{2}$ with CLAS12. The plans for the preparation of this experiment recently approved (JLab PAC44) experimental program with the CLAS12 were presented in the talk by L. Lanza (INFN). The three other approved experiments aimed toward obtaining the $\gamma_{\mathrm{v}} \mathrm{NN}^{*}$ electrocouplings of most $\mathrm{N}^{*}$ states in mass range up to $3.0 \mathrm{GeV}$ and at $2.0 \mathrm{GeV}^{2}<\mathrm{Q}^{2}<12 \mathrm{GeV}^{2}$ from exclusive $\mathrm{N} \pi, \pi^{+} \pi^{-} \mathrm{p}$, and KY electroproduction data will start in the first run period in 2017 and possibly 2018. Further development of the reaction models incorporating the quark degrees of freedom is urgently needed to facilitate the extraction of the resonance electrocouplings at high $\mathrm{Q}^{2}$. The prospects for the development of such an approach were outlined by P. Kroll (University of Wuppertal and University of Regensburg).

The resonance electroexcitation will be explored in these experiments at the distances where the quark core dominates, for the first time offering direct access to dressed quarks and their non-perturbative strong interaction. The dressed quark mass function will be probed at the distance scales where the transition from the quark-gluon confinement to $\mathrm{pQCD}$ regimes takes place, addressing the most challenging and still open problems of the Standard Model on the nature of the dominant part of the hadron mass, quark-gluon confinement, and the emergence of hadrons from QCD.

The reader can find more details about the mentioned presentations and many other interesting contributions in this peer-reviewed special issue of these Proceedings. The detailed program and all the presentations can be found in: http://boson.physics.sc.edu/ gothe/ect*-15/program.html. 